\title{
Exploring Solar System Organic Chemistry Evolution through the Surfaces of Ceres and Large Asteroids
}

Marc Neveu, Department of Astronomy, University of Maryland, College Park, MD and Planetary Environments Laboratory, NASA Goddard Space Flight Center, Greenbelt, MD.

\section{Co-authors:}

José C. Aponte, Astrochemistry Laboratory, NASA Goddard Space Flight Center, Greenbelt, Maryland and Department of Chemistry, The Catholic University of America, Washington D. C. Julie Castillo-Rogez, Jet Propulsion Laboratory, California Institute of Technology, Pasadena, $C A$.

Bethany L. Ehlmann, California Institute of Technology, Pasadena, CA

Heather B. Franz, Planetary Geology, Geophysics, and Geochemistry Laboratory, NASA Goddard Space Flight Center, Greenbelt, MD.

Christopher H. House, Department of Geosciences and Earth and Environmental Sciences Institute, The Pennsylvania State University, University Park, PA.

Erwan Mazarico, Planetary Geology, Geophysics, and Geochemistry Laboratory, NASA Goddard Space Flight Center, Greenbelt, MD.

Alex Pavlov, Planetary Environments Laboratory, NASA Goddard Space Flight Center, Greenbelt, $M D$

Vassilissa Vinogradoff, CNRS, Aix-Marseille University, PIIM, LAM, France.

Kris Zacny, Exploration Technologies, Honeybee Robotics, 2408 Lincoln Ave, Altadena, CA. 
Key points:

- Accessing and characterizing solar system organic material is crucial for understanding the processes by which our Solar System became habitable.

- Recently exposed surfaces in craters of large asteroids (e.g., 10 Hygiea, 24 Themis, Ceres), with a large diversity of ages and abundant organic compounds, provide a natural laboratory to study Solar System organic chemistry and the potential for habitability throughout time. For the dwarf planet Ceres, surface exploration is possible at Discovery and New Frontiers costs. Ceres has likely hosted habitable environments, possibly prebiotic ingredients, and perhaps even incipient life at some time in its history.

- Ceres' organic material is abundant enough to be seen from orbit. Ceres' surface spans (1) geologically old areas comprising $\sim 10 \mathrm{wt}$. \%t carbon, (2) a few locations with higher concentrations of more diverse organic compounds, and (3) bright areas (mostly sodium carbonate), some at most a few $10^{5}-10^{6}$ years old and perhaps much younger. This young surface age is an opportunity to examine organic matter evolution, including both products recently exposed from Ceres' interior and those modified by radiolysis damage.

- Radiative processing has been a major factor shaping organic evolution throughout Solar System history, both degrading compounds (e.g., molecular biomarkers) and increasing organic complexity. Laboratory investigations of both aspects, including on products of aqueous reactions, are needed to advance our understanding of habitable worlds.

\section{Follow the organics}

Organic material enables life. Accessing and characterizing solar system organic material is key to understanding the processes by which Solar System worlds became habitable and, for some, inhabited. Understanding organic evolution from Solar System formation to the present requires identifying precursor molecules and elucidating pathways of synthesis and modification.

Chief among these are photo- and radiolytic processes and aqueous alteration or metamorphism (Chang \& Bunch 1986; Shock \& Schulte 1990; Aponte et al. 2017), which produce organic compounds relevant to prebiotic chemistry. Solar radiation was the largest energy source in the young Solar System, and affected organic matter before accretion in planetesimals and then on the surfaces of planetary bodies. Organic material synthesized and modified by radiolysis, photolysis, or photoexcitation, further reacted in planetary bodies through the interaction of water and rock fueled by radiogenic and impact heat.

\section{Ceres and like asteroids, record keepers of organic evolution}

Understanding processes that drive the synthesis and evolution of organic material that contributes to making worlds habitable requires leaving Earth. On our planet, the processes that led to the organic makeup that preceded the emergence of life but paved the way for it have been hopelessly difficult to disentangle from the imprint of biology. Arguably, the worlds best suited for investigating abiotic organic evolution are those on which such organic synthesis is ongoing (to minimize biases introduced by subsequent modification or destruction processes) and where 
organic compounds are abundant (to ensure detection and characterization of a broad range of compounds, which helps elucidate synthesis pathways and the full suite of products). Although organic synthesis can proceed in atmospheres, including likely that of early Earth, the level of chemical complexity that led to life likely required additional endogenic processing of compounds predating accretion or photochemical products in liquid water.

Very few accessible (e.g., surface or plume) solar system locales meet all three criteria (Fig. 1), in part because they act against one another. For example, Earth's ongoing endogenic abiotic organic synthesis feeds life; its consumption results in a small proportion of abiotic organic compounds. A record of solar system organic matter is preserved, albeit likely degraded, in carbonaceous chondrites, but less-modified products of recent syntheses are in all likelihood absent. All three conditions are likely met in the interiors of ocean worlds but masked by atmosphere-surface physical and chemical interactions or a thick, diluting hydrosphere. Large

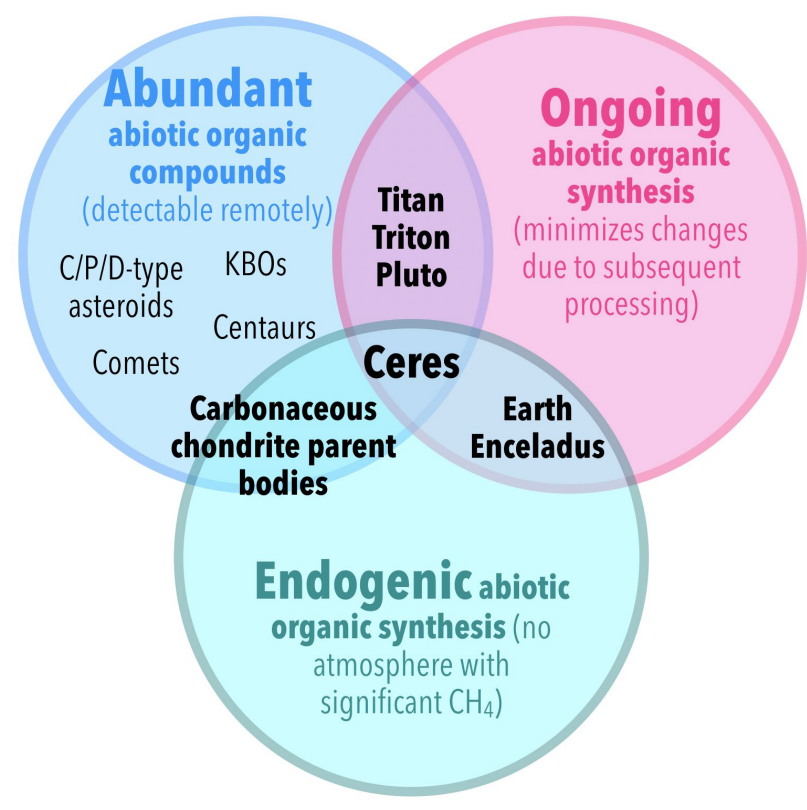
protoplanets, the survivors of those that contributed to delivering water and organic material to the terrestrial planets, have organic material that is both endogenous and abundant. Among carbonaceous protoplanets, Ceres is the best-known representative and perhaps the only one both with a record of ancient organic material and where endogenic synthesis might continue today, as detailed below.

Figure 1. Large asteroids such as Ceres are the only solar system bodies preserving a record of organic synthesis spanning both early solar system material and the present day. They may be our only window into solar system organic chemistry evolution.

Surface regions on Ceres span ages from the early solar system to the present day:

- Most of the dark surface (Fig. 2a) was likely emplaced early in solar system history and contains up to $\mathbf{2 0} \%$ carbon of organic origin. Its mineralogy "appears to be dominated by products of rock-fluid interactions, such as phyllosilicates - some of which are $\mathrm{NH}_{4}$-bearingand carbonates. [...] Ceres's mineral and elemental data can be explained by the presence of carbonaceous chondritic-like materials ( 50-60 vol\%), possibly due to infalling asteroids, admixed with aqueously altered endogenic materials that contain higher-than-chondritic concentrations of carbon. [...] Ceres's surface may contain up to $20 \mathrm{wt} \%$ of carbon, which is more than five times higher than in carbonaceous chondrites. The coexistence of phyllosilicates, magnetite, carbonates and a high carbon content implies rock-water alteration played an important role in promoting widespread carbon chemistry” (Marchi et al. 2019). The overall homogeneity of Ceres' surface suggests a global emplacement at an early time when liquid water was ubiquitous in Ceres' interior, which likely required heating by short-lived radionuclides in 
the first few million years (e.g., Neveu and Desch 2015; Ammannito et al. 2016; Travis et al. 2018; Castillo-Rogez et al. 2019).

\section{- Select areas of the surface appear geologically very recent and likely bear organic} material. Examples are the dome of Cerealia facula in the center of Occator crater (Fig. 2b) as well as other bright, recently exposed areas in Haulani and Oxo craters, and others. Some of these areas are at most a few $10^{5}-10^{6}$ years old (Nathues et al. 2017; Krohn et al. 2018; Scully et al. 2018) and perhaps much younger. These areas are rich in sodium- and ammonium-carbonate salts and chlorides (De Sanctis et al. 2020), and some bear water ice which is unstable below $\sim 50^{\circ}$ latitude on Ceres (Combe et al. 2016). Predicted sub-percent abundances of organic compounds (Neveu et al. 2017; Castillo-Rogez et al. 2018) are too low for remote detection but copious for in situ or sample return investigations, as demonstrated at Enceladus (e.g., Postberg et al. 2018). The present-day subsurface aqueous environments in which endogenic synthesis may be taking place (Hesse \& Castillo-Rogez 2019; Ruesch et al. 2019) may be kept liquid by radiogenic or impact heating.

Figure 2. Ceres records billion-year-old organic synthesis in the presence of liquid water on most of its surface (a). Products of ongoing synthesis may be available at select areas such as Cerealia facula in Occator (b). Even measurements made from orbit distinguish different types of organic compounds at the surface, such as aliphatic species at Ernutet crater (c).

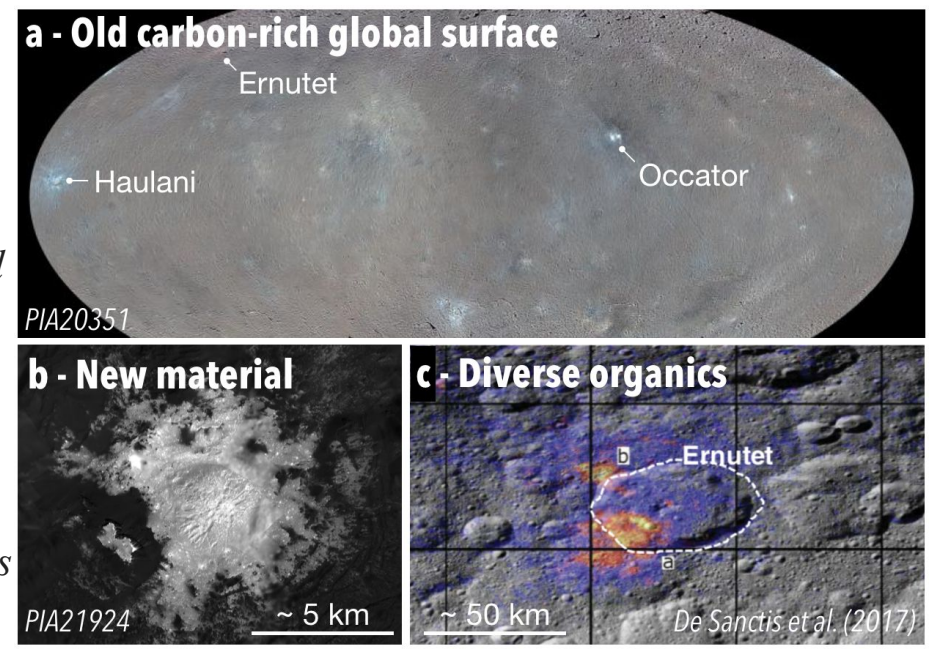

- Other areas of undetermined age are organic hot spots. Ernutet crater, despite not showing any outstanding morphology, is the site of a patch rich in aliphatic (chained) and possibly aromatic (ringed) organic compounds that straddles part of its rim (De Sanctis et al. 2017, 2019). This is surprising because organic compounds tend to degrade into 6-carbon ring structures (ultimately, graphite), and/or become disordered (amorphous) carbon due to space weathering (see next section). The presence of not fully aromatic or disordered organic carbon seen from orbit thus suggests fresh sourcing from recent excavation from the subsurface where these compounds were synthesized (Bowling et al. 2020). Depending on the structure and composition of these aliphatic compounds, maximum abundances at Ernutet range between 5 area\% and 65 area\% (Kaplan et al. 2018); recent results suggest 15 wt.\% (Vinogradoff et al. 2019).

Other protoplanets, too (Fig. 3). Although Ceres is the only large carbonaceous airless world studied in detail, it may not be the only host of abundant endogenic organic material with ages spanning solar system history. The third and fourth largest main-belt objects, Pallas and Hygiea, also have surface compositions (inferred from remote measurements) reminiscent of carbonaceous chondrites. These $500 \mathrm{~km}$-class objects are highly unlikely to preserve any liquid by radiogenic heating alone (since even Ceres is at best on the brink of being fully frozen; Neveu and Desch 2015; Travis et al. 2018; Castillo-Rogez et al. 2019), so any geologically recent or 
current organic synthesis on these bodies hinges on their recent bombardment by impactors able to generate local melting. However, the absence of bright spots in best-resolution images (at which Occator faculae are obvious; Vernazza et al. 2020) of Pallas and Hygiea does not bode as well for the presence of recently synthesized organic compounds. On the other hand, the existence of collisional families for both Pallas (Lemaître \& Morbidelli 1994) and Hygiea (Zappalà et al. 1995) offers the prospect of sampling (old) material from their interior. These objects and others (e.g., the 200-km Themis) show compositional variations relative to Ceres (Larson et al. 1983; Campins et al. 2010; Rivkin \& Emery 2010; Vernazza et al. 2017; Rivkin et al. 2019; see white paper by Landis et al.). Such diversity in carbonaceous planetesimal compositions would help inform the diversity of conditions in early organic synthesis beyond the information provided by carbonaceous meteorites (e.g., within geologic context).

Figure 3. Spatially resolved images and spectra of the largest protoplanets acquired in the past decade have enabled the identification of prime locations for investigating the origin and fate of organic compounds that make worlds habitable. Ceres image obtained by the Dawn mission; Pallas and Hygiea images

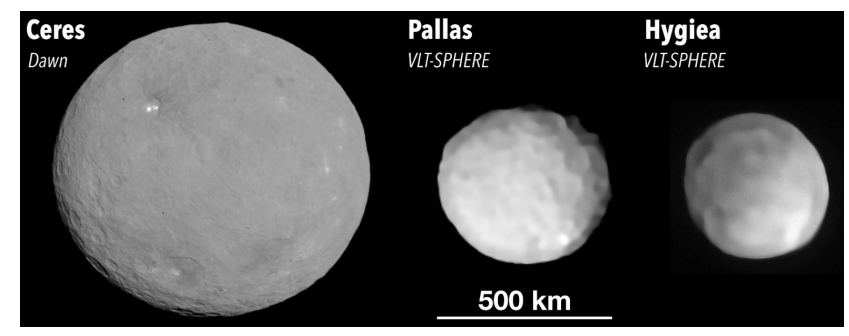
obtained by Vernazza et al.

Sampling a single versus multiple sites. At the sensitivity and spatial resolution of Dawn measurements, areas of Ceres' surface with newly synthesized compounds (e.g., Occator's faculae) and enriched in organics (e.g., Ernutet) are far apart, though the ubiquitous regolith at all locations contains ancient organic material at abundances greater than carbonaceous chondrites. Mobility on Ceres is expensive in terms of propellant but hopping between sites separated by tens of $\mathrm{km}$ is feasible within the New Frontiers cost cap (Castillo-Rogez et al. PMCS report).

Similarly, sampling carbonaceous protoplanet compositions that differ from Ceres' may require visiting other objects. This brings about a trade between the diversity of organic syntheses that can be accessed and the scope of the mission(s) required to access it. In rough order of increasing science return and increasing cost, possible options include:

- Multiple remote-sensing missions to protoplanets other than Ceres (Discovery-class), provided evidence for geologically seemingly ancient and recent organic material can be uncovered with upcoming telescopes (e.g., extremely large ground-based telescopes and JWST). The quality of the data obtained on such objects even with existing assets (Rivkin et al. 2019; Vernazza et al. 2020; Fig. 3) suggests the potential of next-generation telescopes to obtain such evidence.

- A single-site in situ investigation at Ceres (Discovery-class) with the capability to determine the organic matter composition of one or more compositional unit(s) of Ceres' surface.

- A mobile in situ investigation at Ceres (New Frontiers-class, see PMCS report) with the ability to carry out detailed organic matter compositional analysis.

- Sample return from Ceres (New Frontiers-class, see PMCS report). The PMCS report features sampling from a single site in fresh evaporites but additional sites may be feasible.

Where the "knee in the curve" lies in science return per dollar remains to be demonstrated by mission concept studies. The Ceres PMCS report provides essential input in this regard, as well as in the types of measurements required and the spaceflight readiness of measurement methods. 


\section{Radiation processing of surface organic material}

Today, water-rock interaction has all but ceased but its organic products exposed at asteroidal surfaces continue to undergo radiative processing. Disentangling radiation processing post-surface emplacement from prior drivers of organic evolution presents a challenge to understanding the emergence and persistence of habitable environments in the Solar System.

Radiation giveth organic material. Organic compounds can be formed from carbon-bearing ices (e.g., $\mathrm{CO}, \mathrm{CO}_{2}, \mathrm{CH}_{4}, \mathrm{HCN}$ ) through chemical reactions driven by radiolysis or $\mathrm{UV}$ photolysis. This has been studied most extensively for extremely cold environments, such as comet-forming regions of the Solar System, molecular clouds, and the interstellar medium (e.g., Takano et al. 2007; Gontareva et al. 2009; Hudson and Loeffler 2013; Bergantini et al. 2017; Bibang et al. 2019). Radiolysis by galactic cosmic rays may have formed organic material at the dark poles of the Moon and Mercury (Crites et al. 2013). In addition, complex biochemical compounds have been synthesized experimentally from UV irradiation of precursor molecules in the presence of different mineral samples relevant to the surfaces of small bodies in the Solar System (Simakov \& Kuzicheva 2005).

Radiation taketh organic material away. Ion irradiation of carbonaceous material analogous to interstellar materials clearly causes destruction of aliphatic $\mathrm{C}-\mathrm{H}$ features over time (Godard et al. 2011). An application to Ceres is shown in Fig. 4, in which experimental results on amino acid degradation by $\gamma$-ray irradiation are combined with estimates of radiation fluxes at Ceres to calculate the fraction of molecule survival over time. Preservation is increased at a few cm depth because by $5 \mathrm{~cm}$, most solar irradiation is absorbed, while galactic cosmic radiation (GCR) decreases slowly between 5 and $10 \mathrm{~cm}$. These results suggest that abundances of key organic compounds such as amino acids, if emplaced within a few $\mathrm{cm}$ of Ceres' surface in ice-poor regolith, should remain within the same order of magnitude for tens of millions of years; i.e., not significantly changed (relative to the limits of detection of measurement techniques) in recent surface locations such as Occator crater where time scales preclude significant impact gardening. They also show that the mineralogy in which organic compounds can be embedded can significantly affect their survival; experiments to date have only scratched the surface in exploring this essential parameter space.

Figure 4. Radiation

degradation of the

medium-mass amino acid isovaline (117 amu) in two endmember cases at conditions approximating the environment of Occater crater on Ceres: $2.7 \mathrm{AU}$ from the Sun, temperature $\approx$ $220 \mathrm{~K}$ (local noon; Hayne \& Aharonson 2015), surface density $1.3 \mathrm{~g} \mathrm{~cm}^{-3}$ (Ermakov et al. 2017), exposure age 1.3-64 Myr (Neesemann et
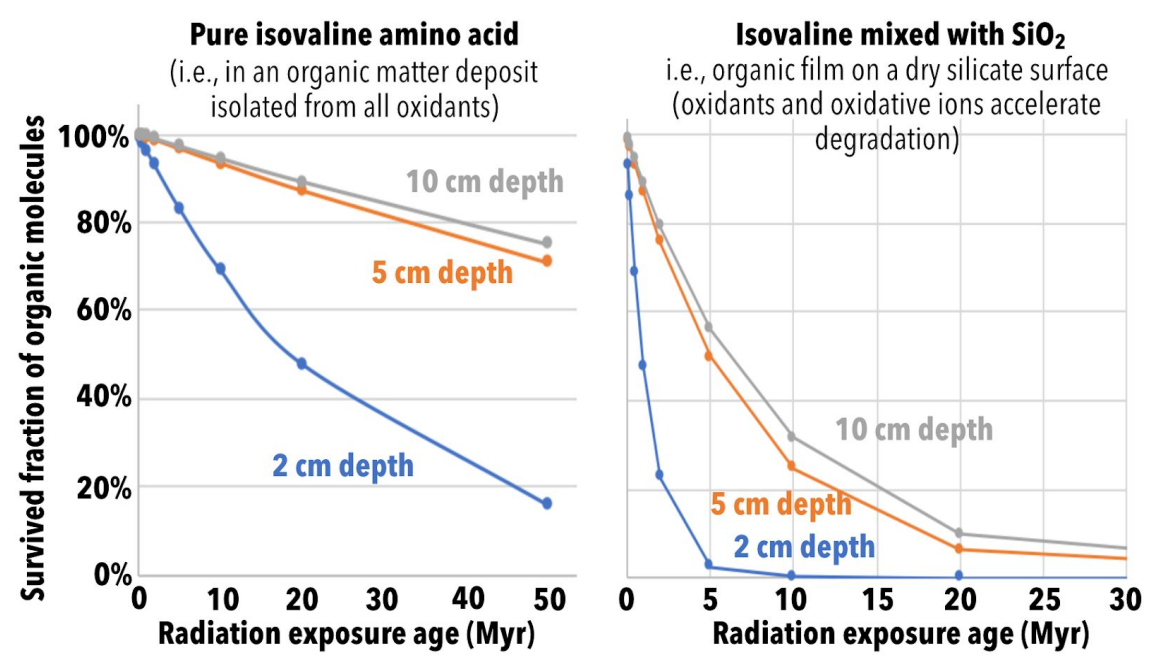
al. 2019). Smaller amino acids degrade more slowly. Average solar and GCR spectra from Pavlov et al. $(2012,2014)$; the GCR flux was increased by 10\% compared to the flux at $1 \mathrm{AU}$ due to less modulation by the heliosphere. No water was assumed; for organic molecules embedded in ice, degradation would be faster. The experimental radiolysis data used were obtained from $\gamma$-ray irradiation; protons tend to increase degradation for the same dosage. These data are for local noon temperatures; degradation is expected to be substantially slower at Occator's $\approx 160$ K average diurnal temperatures (Hayne \& Aharonson 2015).

\section{Reconstructing the history of organic material: experiments needed}

Compositional and contextual measurements of surface organic material are underpinned by observational techniques of astronomy and geology, but reconstructing the formation and evolution of organic material also requires experimental chemistry. Even when in situ or sample return investigations establish the concentration and molecular distribution of organic matter, experimental irradiation and hydrothermal processing of material analogous to that inferred to be present on Ceres and like asteroids is needed to firmly assess the origin of such compounds.

Example notable experimental results. While not fully representative of asteroid or Ceres surfaces, irradiation of carbonaceous chondrites produces spectral variations in the visible and near-infrared with inverse reddening/darkening trends for objects rich or poor in carbon (Lantz et al. 2015, Brunetto et al., 2018). This provides a means to constrain the exposure age of planetary surfaces by spectroscopy. In experiments simulating the inner structure of icy dust grains exposed to high-energy radiation, polypeptides were formed from single amino acids (Gontareva et al. 2009). Past aqueous alteration experiments have produced hundreds of molecules of a given compound starting from one precursor (Cody et al. 2011; Vinogradoff et al. 2018; Isono et al. 2019), leading to sequestration of organic molecules inside phyllosilicates (at least 7\%) (Vinogradoff et al. 2020), to the production of insoluble polymeric carbon structures (Kebuwaka et al. 2013), and amino acids (Kebuwaka et al. 2017).

The need for more analog experiments. A valuable attempt to reconstruct the early and current organic content observed on worlds such as Ceres requires carrying out systematic studies of the formation of organic compounds through a combination of irradiation and hydrothermal processing experiments. Since organic matter can be intimately mixed with minerals (typically phyllosilicates but also salts), as observed both in carbonaceous chondrites and on Ceres, we recommend scrutinizing the effect of minerals (e.g., ammonium phyllosilicates and salts in the case of Ceres) on the survivability of organic matter on irradiated planetary surfaces.

An example avenue would involve these steps: (1) simulate icy grains under conditions relevant to dense molecular clouds and protoplanetary disks, followed by (2) continued synthesis and modification of organic mixtures via hydrothermal processes post-accretion, and (3) re-irradiate to mimic the expected time a given surface has been exposed. An alternative avenue could involve continued irradiation at surface conditions in the presence of realistic analogous mineralogy. Generalizing experimental results to predict organic modification for different ice and mineral compositions, radiation doses, and hydrothermal conditions (e.g., temperatures) requires experimental modeling (Fig. 4).

Isotopic clues. The stable isotopic composition of organic material represents another key component in understanding its origin and history. Analyses of organic compounds in chondrites 
have revealed a wide range of values in isotope ratios, particularly those of $\mathrm{H}, \mathrm{C}, \mathrm{N}$, and $\mathrm{O}$ (e.g., Kerridge 1985; Elsila et al. 2012), which often differ from those typical of terrestrial biomarkers. This observation is helpful in identifying products of abiotic organic chemistry on the meteorite parent bodies by screening out terrestrial contamination. Stable isotopes also offer a means to test hypotheses concerning the origins and modification histories of organic compounds and water on Ceres, e.g., whether the volatile inventory represents a single source incorporated during primary accretion or whether some compounds are derived from materials delivered to the surface later, such as by comet impacts. Stable isotope ratios should be key measurements for in situ missions to and sample return from Ceres (see white paper by Miller et al.). Incorporating isotopic measurements in the above supporting experimental avenues is thus essential.

Understanding the processes by which Solar System worlds became habitable requires exploration of Ceres (and like asteroids) and organic chemistry experiments essential to the interpretation of mission measurements.

References: Ammannito et al. (2017) Science 353, aaf4279. Aponte et al. (2017) ACS Earth \& Space Chem 1, 3. • Bibang et al. (2019) Low Temp. Phys. 45, 590. • Bowling et al. (2020) EPSL 534, 116069. • Brunetto et al. (2018) PSS 158, 38. • Campins et al. (2010) Nature 464. 1320. • Castillo-Rogez et al. (2018) MaPS 53, 1820. • Castillo-Rogez et al. (2019) GRL 46, 1963. • Castillo-Rogez et al. (2020) Astrobiology 20, 269.・Chang \& Bunch (1986) In Clay Minerals and the Origin of Life, p. 116-129. C Cody et al. (2011) PNAS 108, 19171. • Combe et al. (2016) Science 353, aaf3010. • Crites et al. (2013) Icarus 226, 1192. • De Sanctis et al. (2017) Science 355, 719. • De Sanctis et al. (2019) MNRAS 482:2407. • De Sanctis et al. (2020) Nat Astron, avail. online Aug. $10 \bullet$ Elsila et al. (2012) MaPS 47, 1517. • Engel (2013) Proc SPIE $\underline{8865,886505}$. Ermakov et al. (2017) JGR Planets 122, 2267. • Gontareva et al. (2009) PSS 57, 441. • Hayne \& Aharonson (2015) JGR Planets 120, 1567. • Hesse \& Castillo-Rogez (2019) GRL 46, 1213. - Kaplan et al. (2018) GRL 45, 5274. - Kebukawa et al. (2013) ApJ 771, 19. - Kebukawa et al. (2017) Sci Adv 3, e1602093. - Kerridge (1985) GCA 49, 1707. - Krohn et al. (2018) Icarus 316, 84. • Lantz et al. (2015) A\&A 577, A41. • Larson et al. (1983) Icarus 56, 398. - Lemaitre \& Morbidelli (1994) Cel Mech Dyn Astron 60, 29. • Marchi et al. (2019) Nat Astron

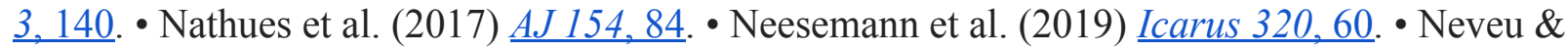
Desch (2015) GRL 42, 10197. • Neveu et al. (2017) GCA 212,324. • Pavlov et al. (2012) GRL 39, L13202. • Pavlov et al. (2014) JGR Planets 119, 1390. • Postberg et al. (2018) In Enceladus and the Icy Moons of Saturn, p.129-162. • Rivkin \& Emery (2010) Nature 464, 1322. • Rivkin et al. (2019) JGR Planets 124:1393. • Ruesch et al. (2019) Nat Geo 12, 505. - Scully et al. (2019)

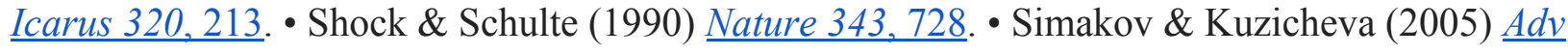
Sp Res 36, 190. • Takano et al. (2007) EPSL 254, 106. • Travis et al. (2018) MaPS 53, 2008. • Vernazza et al. (2017) AJ 153, 72. • Vernazza et al. (2020) Nat Astron 4, 136. • Vinogradoff et al. (2018) Icarus 305, 358. • Vinogradoff et al. (2019) EPSC-DPS abstract 690-1. • Vinogradoff et al. (2020) GCA 269,150. • Zappalà et al. (1995) $\underline{\text { Icarus 116, } 291 .}$ 